\title{
The Influence of the Intermittent Behavior of the Nocturnal Atmospheric Flow on the Prediction of the Diurnal Temperature Range: A Simplified Model Analysis
}

\author{
Leandro L. Gonzales, ${ }^{1,2}$ Felipe D. Costa, ${ }^{2}$ Otávio C. Acevedo, ${ }^{3}$ Daniel M. dos Santos, ${ }^{2}$ \\ Luiz E. Medeiros, ${ }^{2}$ Arlindo D. Carvalho Junior, ${ }^{2}$ Franciano S. Puhales, ${ }^{3}$ Vagner Anabor, ${ }^{3}$ \\ Luis G. N. Martins, ${ }^{3}$ Giuliano Demarco, ${ }^{4}$ and Gervásio A. Degrazia ${ }^{3}$ \\ ${ }^{1}$ Instituto Federal Sul-Rio-Grandense, Campus Pelotas, Pelotas, RS, Brazil \\ ${ }^{2}$ Programa de Pós-Graduação em Engenharia, Universidade Federal do Pampa, Campus Alegrete, Alegrete, RS, Brazil \\ ${ }^{3}$ Departamento de Física, Universidade Federal de Santa Maria, Santa Maria, RS, Brazil \\ ${ }^{4}$ Departamento de Engenharia Mecânica, Universidade Federal de Santa Maria, Santa Maria, RS, Brazil \\ Correspondence should be addressed to Felipe D. Costa; fdenardin@unipampa.edu.br
}

Received 1 February 2017; Revised 17 April 2017; Accepted 2 May 2017; Published 8 June 2017

Academic Editor: Enrico Ferrero

Copyright (C) 2017 Leandro L. Gonzales et al. This is an open access article distributed under the Creative Commons Attribution License, which permits unrestricted use, distribution, and reproduction in any medium, provided the original work is properly cited.

\begin{abstract}
The variation of the atmospheric temperature near the surface associated with anthropogenic effects is analyzed using a simplified atmospheric model. Local changes in cloud cover and four different scenarios of atmospheric concentration of carbon dioxide are considered. The results show that the highest temperature variability occurs in the weak wind and decoupled state and in the transition between flow regimes. In agreement with previous efforts, the results indicate that the reduction of diurnal temperature range is related to the existence of two distinct flow regimes in the stable boundary layer. However, in the decoupled state, the occurrence of intermittent bursts of turbulence may cause temperature variations among the different scenarios to become unpredictable. It implies that it is difficult to predict the diurnal temperature range in places where low winds are common.
\end{abstract}

\section{Introduction}

The increase of the concentration of greenhouse gases, such as methane and $\mathrm{CO}_{2}$, in the atmosphere, has been causing anomalous elevations in the temperature in the last two centuries [1]. Furthermore, the temperature elevation is not homogenous throughout the diurnal cycle. It has been suggested that the daily minimum temperatures may have risen twice as fast as the corresponding maxima since $1950[2,3]$. Those observations, however, are not generally reproduced by atmospheric models $[4,5]$. Reference [4] observed that doubling the concentration of the carbon dioxide was not enough to significantly affect the diurnal temperature range (DTR). Reference [5] found similar results and suggested that the problem resides in the turbulence boundary layer schemes that are used in the atmospheric models, mainly because of their bad performance during nighttime.
In this period, the only source of turbulence is the wind shear, as the stable stratification causes buoyant forces to destroy turbulence. The relative magnitude of those two terms from the turbulent kinetic energy budget equation ultimately leads to two distinct regimes in stable boundary layer (SBL) [6-9]. A decoupled regime is characterized by light winds and weak turbulence that can alternate with periods with more intense turbulence, in a phenomena known as global intermittency, or just intermittency [6]. On the other hand, the coupled state happens for moderate to strong winds, and the turbulence is always well developed and self-sustained [10]. During the same night, the flow can switch regimes, and those changes can occur intermittently or in an organized way and can lead to temperature variability as large as $10 \mathrm{~K}$ [11]. Recent studies [8-10] have identified that to a large extent the SBL regime is solely determined by mean wind speed. Anyhow, atmospheric models do not usually represent well 
the SBL variability of regimes, and it is pointed out as one of the causes of the unsuccessful prediction of the DTR behavior [12].

Reference [13] presented, by using the numerical model proposed by [14], an analysis of the influence of the atmospheric coupling in the nocturnal temperature and its possible impact over the DTR. In this work we use a similar model, proposed by [15], which is able to reproduce the intermittent behavior of the turbulence in the disconnected state, to analyze the impact of the complex behavior of the turbulence over the temperature estimations. It is important to stress that the analysis presented here is simplified in many ways. For example, the model does not take into account the effect of the radiative flux divergence, which exerts partial control over the stability of the layer, especially in weak wind cases $[16,17]$. However, it can illustrate the impact of a phenomenon that most of the atmospheric models are not able to reproduce on the DTR. And, it also shows that many additional efforts are necessary to improve such models.

\section{Materials and Methods}

2.1. Model. For idealized conditions, with no advection, and for a dry and horizontally homogeneous atmosphere, the equations that control the flow in the atmospheric boundary layer $(\mathrm{ABL})$ can be written in simplified form as

$$
\begin{aligned}
& \frac{\partial u}{\partial t}=f\left(v-v_{G}\right)-\frac{\partial\left(\overline{u^{\prime} w^{\prime}}\right)}{\partial z}, \\
& \frac{\partial v}{\partial t}=f\left(u_{G}-u\right)-\frac{\partial\left(\overline{v^{\prime} w^{\prime}}\right)}{\partial z}, \\
& \frac{\partial \theta}{\partial t}=-\frac{\partial \overline{w^{\prime} \theta^{\prime}}}{\partial z},
\end{aligned}
$$

where $u, v$, and $\theta$ are, respectively, the wind components and the air temperature, $u_{G}, v_{G}$ are the components of the geostrophic wind, $\overline{u^{\prime} w^{\prime}}$ and $\overline{v^{\prime} w^{\prime}}$ are the components of the moment flux, and $\overline{w^{\prime} \theta^{\prime}}$ is the sensible heat flux. Following $[12,13]$ the clear air radiative cooling is neglected. Although it is a simplification, as it was stated by [13] and tested here (not shown), the impact of using a simple parametrization for the clear air radiative cooling in the model dynamics is minimal because it does not affect the equilibrium states of the model.

2.1.1. Surface Parameterization. The surface parameterization is an important aspect to determine the nature and the behavior of the boundary layer. In this work a method proposed by [18] is used, which considers the energy balance of the soil substrate, a slab layer of soil, and the surface atmospheric layer $[14,18]$. In this way, the prognostic equation for the soil temperature can be written as

$$
\frac{\partial \theta_{g}}{\partial t}=\frac{1}{C_{g}}\left(I_{\downarrow}-\sigma \theta_{g}^{4}-H_{0}\right)-k_{m}\left(\theta_{g}-\theta_{m}\right),
$$

where $I_{\downarrow}$ is the flux incoming longwave radiation, $\theta_{g}$ is the ground temperature, $\theta_{m}$ is the substrate temperature, $H_{0}$ is the surface sensible heat flux, $k_{m}$ is the heat exchange coefficient between the substrate and the slab, and $\sigma$ is the Stefan-Boltzmann constant. The thermal capacity per area unit of the surface $C_{g}\left(\mathrm{JK} \mathrm{m}^{2}\right)$ depends on the soil thermal conductivity $\lambda$, the Earth angular frequency $\omega$, and the soil volumetric thermal capacity $C_{s}=c_{s} \rho_{s}$, where $c_{s}$ and $\rho_{s}$ are the soil specific heat and density, respectively [18]:

$$
C_{g}=0.95\left(\frac{\lambda C_{s}}{2 \omega}\right)^{1 / 2} \text {. }
$$

2.1.2. Radiative Balance. The radiative forcing related to a perturbation in the concentration of a gas is defined by the net change of the radiative flux induced in the Tropopause, which is generally recognized as a gain (positive) or loss (negative) of energy to the whole system. The justification for this concept rises from experiments with radiative-convective one-dimensional model, in which the temperature surface changes can be only related to the net changes of the radiative flux in the Tropopause [19].

In a situation of clear skies, the surface radiative flux is, for convenience, interpreted in terms of the definition of effective atmospheric emissivity, $\varepsilon_{a}$, given by the nondimensional relation $\varepsilon_{a}=I_{\downarrow} /\left(\sigma \theta_{0}^{4}\right)$, where $\theta_{0}$ is the temperature in the lower limit of the atmosphere. According to [20], the incoming longwave radiation is given by

$$
I_{\downarrow}=\sigma\left(Q_{c}+0.67\left(1-Q_{c}\right)\left(1670 Q_{a}\right)^{0,05}\right) \Theta^{4}+\text { GHGS, }
$$

where $Q_{c}$ is the cloud fraction, $Q_{a}$ is the specific humidity, and $\Theta$ is the temperature in a reference level. It is important to notice that the term GHGS is added due to greenhouse gas concentration [13], and it defines the radiative forcing due to the carbon dioxide concentration in the atmosphere [21]:

$$
\mathrm{GHGS}=6,3 \ln \left(\frac{C}{C_{0}}\right),
$$

where $C_{0}$ corresponds to current values of $\mathrm{CO}_{2}$ in the atmosphere.

2.1.3. Turbulence Closure. Usually, simplified models use the flux-gradient theory to solve the turbulence closure problem. When a first-order closure is used, those models use prescribed stability functions on the diffusion coefficients, or on the mixing lengths, which reduces the degrees of freedom of a dynamical system. Then, a possible complex relation between two variables is replaced by mean relation that may "kill" any possibility of the model to reproduce complex solutions. In this way [15] proposed not to use any stability function. In this way, in the whole boundary layer the turbulent fluxes are determined by

$$
\begin{aligned}
-\overline{u^{\prime} w^{\prime}} & =u_{*}^{2} \cos \psi, \\
-\overline{v^{\prime} w^{\prime}} & =u_{*}^{2} \sin \psi, \\
-\overline{w^{\prime} \theta^{\prime}} & =u_{*} \theta_{*},
\end{aligned}
$$


where $\psi=\arctan [(\partial v / \partial z) /(\partial u / \partial z)]$ is the direction of the mean Wind and $u_{*}$ is the friction velocity. Following [22], the friction velocity is directly evaluated from the turbulent kinetic energy (TKE) $\left(u_{*}=\sqrt{E / \alpha}\right)$, with $\alpha$ being a constant that relates the local shear and the turbulence intensity [23]. The value of this constant depends on the local stability; however, for simplicity, we use in this work $\alpha=5.5$ [24].

The temperature scale is defined by

$$
\theta_{*}=\frac{K_{H}(\partial \theta / \partial z)}{u_{*}} .
$$

The diffusion coefficient of heat $\left(K_{H}\right)$ is defined as $K_{H}=$ $K_{M} / \operatorname{Pr}$, where $\mathrm{Pr}$ is the turbulent Prandtl number, assumed to be one for simplicity, and $K_{M}=l_{m} \sqrt{\alpha_{0} E}$ is the diffusion coefficient of momentum. In the latter expression $\alpha_{0}=1 / \alpha$ is a constant and $l_{m}=\kappa z$ is the mixing length, taken as the neutral case to avoid the use of stability functions. It is important to stress that it is a very simplified model in many aspects, and it is necessary to keep the idea of not using stability functions at this point.

A variable that plays a key role in this turbulent closure is the TKE, and for these idealized conditions its budget equation can be written as

$$
\frac{\partial E}{\partial t}=-\overline{u^{\prime} w^{\prime}} \frac{\partial u}{\partial z}-\overline{v^{\prime} w^{\prime}} \frac{\partial v}{\partial z}+\frac{g}{\Theta} \overline{w^{\prime} \theta^{\prime}}-\frac{\partial \overline{w^{\prime} E^{\prime}}}{\partial z}-\varepsilon,
$$

where LHS is the local TKE budget, the two first RHS terms are the shear production terms, the third RHS term is the buoyant production/destruction of TKE, the fourth RHS term is turbulent transport of turbulence, with $\overline{w^{\prime} E^{\prime}}$ being the local turbulent flux of TKE, and the last RHS term is the viscous dissipation of TKE.

Using (6) and (7), and following [25], the following relation can be obtained:

$$
-\overline{u^{\prime} w^{\prime}} \frac{\partial u}{\partial z}-\overline{v^{\prime} w^{\prime}} \frac{\partial v}{\partial z}=\Omega u_{*}^{2}
$$

where $\Omega=\partial U / \partial z$. In the same way the thermal production/destruction of turbulence can be parameterized as

$$
\frac{g}{\Theta} \overline{w^{\prime} \theta^{\prime}}=-R i \Omega u_{*}^{2},
$$

and following [22], the transport term is parameterized as

$$
-\frac{\partial \overline{w^{\prime} E^{\prime}}}{\partial z}=-\left(\frac{K_{M}}{\sigma_{E}} \frac{\partial E}{\partial z}\right)
$$

where $\sigma_{E}$ is a constant that is equivalent to the Prandtl number [22]. Here, this value is taken as 2.5 , the same value used by [15]. The viscous dissipation of TKE is parameterized by the Kolmogorov equation [26, 27]:

$$
\varepsilon=\frac{\left(\alpha_{0} E\right)^{3 / 2}}{l_{\varepsilon}}
$$

In the former expression $l_{\varepsilon}$ is turbulent mixing length for the dissipation, which, for simplicity, is considered to be the same as the turbulent mixing length. It is important to stress that (14) was derivate for conditions of continuous, homogenous, and isotropic turbulence; and such conditions are not present in the very stable regime of the SBL. Then, a constant that represents the presence of anisotropy of turbulence is added to (14). The value of this constant can vary in order of magnitude in models [28]. In this work, we use the same value as [15] $\left(C_{\varepsilon}=0.18\right)$.

Finally, the set of equations that is integrated on the model is

$$
\begin{aligned}
& \frac{\partial u}{\partial t}=f\left(v-v_{G}\right)-\frac{\partial\left(u_{*}^{2} \cos \psi\right)}{\partial z}, \\
& \frac{\partial v}{\partial t}=f\left(u_{G}-u\right)-\frac{\partial\left(u_{*}^{2} \sin \psi\right)}{\partial z}, \\
& \frac{\partial \theta}{\partial t}=-\frac{\partial u_{*} \theta_{*}}{\partial z}, \\
& \frac{\partial E}{\partial t}=\Omega u_{*}^{2}-R i \Omega u_{*}^{2}+\frac{\partial}{\partial z}\left(\frac{K_{M}}{\sigma_{E}} \frac{\partial E}{\partial z}\right)-c_{\varepsilon} \frac{\left(\alpha_{0} E\right)^{3 / 2}}{l_{\varepsilon}}, \\
& \frac{\partial \theta_{g}}{\partial t}=\frac{1}{C_{g}}\left(I_{\downarrow}-\sigma \theta_{g}^{4}-H_{0}\right)-k_{m}\left(\theta_{g}-\theta_{m}\right) .
\end{aligned}
$$

2.1.4. Discretization and Integration. In the model, the top of the boundary layer $(h)$ and the ground surface $(z=0)$ are used as boundaries of the SBL. Between those two points $n$ levels are considered, the first one being fixed at $5 \mathrm{~m}$ and the others being equally spaced between this and the domain top $(h=80 \mathrm{~m})$. The prognostic equation for wind components and temperature is evaluated for these levels. However, the turbulent flux divergence is evaluated where every flux depends on TKE. In this way, the prognostic equation for $E$ is calculated in intermediate levels $\left(z_{i}\right)$ between the main levels $(z)$. The intermediate levels are defined by $z_{i}=\left(z_{i}+\right.$ $\left.z_{i-1}\right) / 2$. So, the set of equations (15) is solved using the lines method, which uses the fourth-order Runge-Kutta as the time integrator, with $0,01 \mathrm{~s}$ for time step.

(1) Initial Conditions and Constants. In the boundary layer's top the variables are assumed as constants: $u(t, h)=u_{G}$, $v(t, h)=v_{G}, \theta(t, h)=\Theta=300 \mathrm{~K}$. In the surface the noslip condition $u(t, 0)=0, v(t, 0)=0$ is assumed. In all levels $v(t=0, z)=0$, while $u(t, z)$ is assumed to increase linearly from its surface value until $u_{G}$. The soil temperature and the air temperature are considered to be the temperature of reference: $\theta_{g}(t=0)=300 \mathrm{~K}$ and $\theta(t=0, z)=300 \mathrm{~K}$.

The initial value of TKE is assumed also as minimum possible value $E$ in all levels, $E(t=0, z)=0.005 \mathrm{~m}^{2} \mathrm{~s}^{-2}$. It is important to notice that it is common to use a minimum value of TKE in atmospheric models [28, 29], but cut-off value does not affect the results as shown by [15]. The other constants used in the model are presented in Table 1. 
TABLE 1: List of constants used in the model.

\begin{tabular}{lcc}
\hline$\omega$ & Earth angular frequency & $7.27 \times 10^{-5} \mathrm{rad} \mathrm{s}^{-1}$ \\
$k_{m}$ & $\begin{array}{c}\text { Heat coefficient transfer from the } \\
\text { substrate to the slab }\end{array}$ & $1.18 \omega$ \\
$\kappa$ & von kármán constant & 0.4 \\
$\mathrm{Pr}$ & Turbulent Prandtl number & 1 \\
$\Theta$ & Reference temperature & $300 \mathrm{~K}$ \\
$\theta_{m}$ & Temperature of the substrate & $285 \mathrm{~K}$ \\
$\rho$ & $\quad$ Air density & $1.225 \mathrm{~kg} \mathrm{~m}^{-3}$ \\
$c_{p}$ & Air specific heat at constant & $1005 \mathrm{~J} \mathrm{~kg}^{-1} \mathrm{~K}^{-1}$ \\
$f$ & pressure & $1 \times 10^{-4} \mathrm{~s}^{-1}\left(\mathrm{for}^{-1}=45^{\circ}\right)$ \\
$Q_{a}$ & Coriolis parameter & $0.03 \mathrm{~g} \mathrm{~kg}^{-1}$ \\
$\sigma$ & Specific humidity & $5.669 \times 10^{-8} \mathrm{~W} \mathrm{~m}^{-2} \mathrm{~K}^{-4}$ \\
$Z_{0}$ & Rtefan-Boltzmann constant & $0.1 \mathrm{~m}^{-1}$ \\
$\lambda$ & Soil thermal conductivity & $0.06 \mathrm{~W} \mathrm{~m}^{-1} \mathrm{~K}^{-1}$ \\
$c_{s}$ & Soil specific heat & $1.92 \times 10^{3} \mathrm{~J} \mathrm{~kg}^{-1} \mathrm{~K}^{-1}$ \\
$\rho_{s}$ & Soil density & $0.30 \times 10^{3} \mathrm{~kg} \mathrm{~m}^{-3}$ \\
\hline
\end{tabular}

\section{Results and Discussion}

Simplified models generally reproduce the bistability of the SBL flow $[14,18,30,31]$; however, to represent the variability of the turbulence in the decoupled state is not an easy task for those models. The model used in this work is able to reproduce both coupling states and the intermittent behavior of the turbulence. Figure 1 shows the time series of temperature at $5 \mathrm{~m}$, for different values of geostrophic wind at the vertical domain top. For $u_{G}=1.0 \mathrm{~m} \mathrm{~s}^{-1}$ (Figure 1(a)), the system is in the decoupled state; however, the solutions are merely periodic, because the mechanical forcing is not strong enough to accelerate the flow to intensify the turbulent activity.

In Figure 1(a) it is also possible to observe that the difference in the temperature can reach $-0.2 \mathrm{~K}$, when the $\mathrm{CO}_{2}$ concentration is reduced to about $27 \%$. On the other hand, when the carbon dioxide concentration doubles, for the same mechanical forcing, the temperature increasing, near the surface, can reach $0.3 \mathrm{~K}$. However, as $u_{G}$ increases the variations on the temperature, for the same concentration of $\mathrm{CO}_{2}$, became unpredictable. It is possible to notice that the dependence of the temperature on the concentration has more or less the same behavior of the dependence on the mechanical forcing; however, it is impossible to estimate correctly the increase in the temperature in the cases of strong intermittency activity (Figures 1(b) and 1(c)). For strong winds (Figure $1(\mathrm{~d})$ ), the system is in the coupled stated and the temperature variability disappears. It is also possible to observe that, for different concentrations, the temperatures do not show significant differences in their values. It is caused by the strong turbulent activity that is sufficient to dissipate or transport the energy in excess near the surface for the upper levels of the atmosphere and so reducing the difference of temperature between the scenarios, as suggested by $[12,13]$.

Figure 2 shows clearly the intermittency influence in the mean temperature for each value of the mechanical forcing.
As the energy that arrives on the surface becomes greater as the carbon dioxide concentration increases, one may think that the temperature also increases. However, in the transition region, between the two regimes the temperature variability is so complex that, even using a $10 \mathrm{~h}$ window to calculate the mean, it is impossible to affirm whether the temperature variation will be positive or negative for little variation in the concentration. In addition, after the connection, the temperature for each concentration could abruptly increase up to $3 \mathrm{~K}$.

A more detailed analysis of how the atmospheric concentration of $\mathrm{CO}_{2}$ affects temperature differences is presented in Figure 3. For values of $u_{G}$ less than $2.0 \mathrm{~m} \mathrm{~s}^{-1}$ the differences in the temperature are well defined, because the occurrence of the turbulence bursts is mainly periodic. During the transition $\left(u_{G}\right.$ between 2.0 and $3.5 \mathrm{~m} \mathrm{~s}^{-1}$ ) the temperature variations turn completely unpredictable, but they oscillate around the mean value that occurs for very weak winds. The major variability occurs during the transition between the two states, where the temperature difference can reach - $1.5 \mathrm{~K}$, in the case when the carbon dioxide concentration is reduced 27\%; on the other hand this difference can be $0.7 \mathrm{~K}$ in a scenario in which the $\mathrm{CO}_{2}$ concentration doubles. This is mainly due the fact that when the system has more energy available, whether mechanical energy or internal energy, the turbulence becomes more intense and as the concentration increases the value of $u_{G}$ necessary for the connection decreases.

Figures 2 and 3 indicate that after the coupling the difference of temperature between the scenarios decreases. Figure 4 shows the dependence of the mean temperature for the different concentrations of $\mathrm{CO}_{2}$ (as Figure 2), after the connection. As expected, according to [12], in the connected state the temperatures differences should be smaller due to the turbulence mixing. In Figure 4 it is possible to observe that the temperature variation, between the actual scenario and the idealized cases where the carbon dioxide concentration is changed, decreases considerably. Just after the connection $\left(u_{G} \approx 3.8 \mathrm{~m} \mathrm{~s}^{-1}\right)$, the temperature difference between all scenarios is about $\pm 0.25 \mathrm{~K}$. However, for a mechanical forcing of $7 \mathrm{~m} \mathrm{~s}^{-1}$ the temperature difference decreases to values near $0.05 \mathrm{~K}$, and it keeps decreasing as the geostrophic wind increases (Figure 4).

One of the major implications of temperature increase is related to the evaporation that will contribute to the cloud formation. The clouds will increase the atmospheric emissivity, so the incoming longwave radiation will increase too. Theoretical studies suggest that the increment in the cloud cover could lead to regional reductions in the DTR between $20 \%$ and 50\%, when compared with clear skies conditions [32, 33]. On the other hand, [33] concludes that the addition in the water vapor on the atmosphere leads to small variations on the DTR, since the diurnal and nocturnal temperature increases are similar.

Here, the analysis in the disconnected state shows that the mean temperature can increase more than $1 \mathrm{~K}$, in the scenario with the actual $\mathrm{CO}_{2}$ concentration, if cloud cover increases just 20\% (figure not shown). However, after the connection the differences are smoothed. Figure 5 shows the 


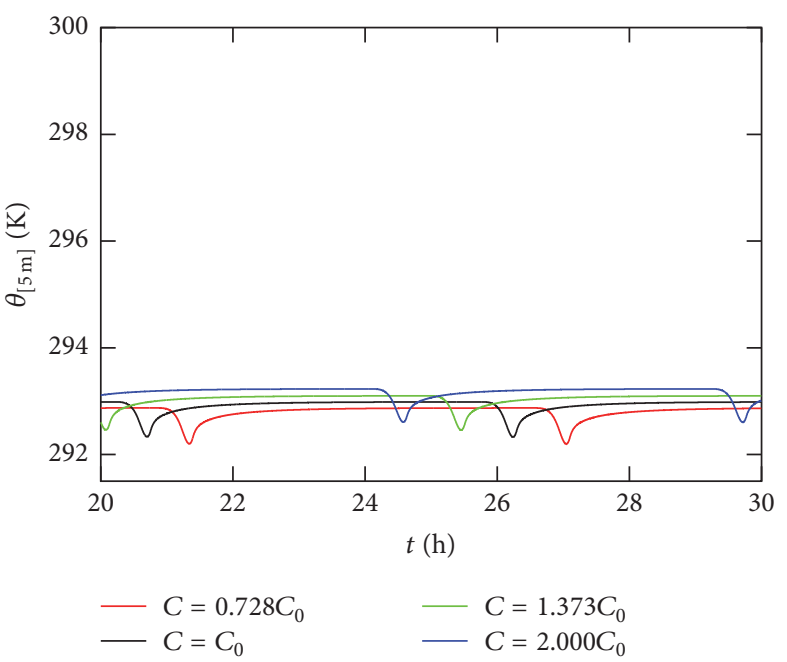

(a) $u_{G}=1.0 \mathrm{~m} \mathrm{~s}^{-1}$

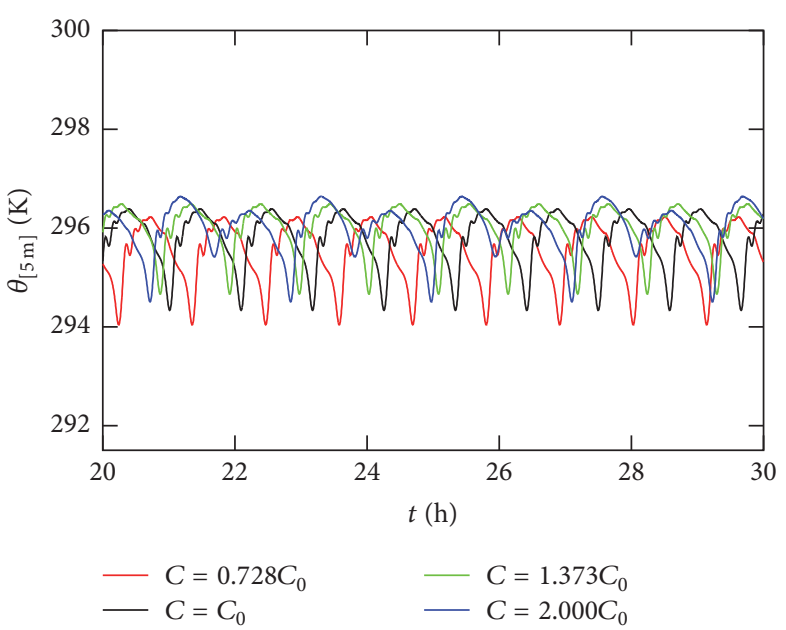

(c) $u_{G}=3.5 \mathrm{~m} \mathrm{~s}^{-1}$

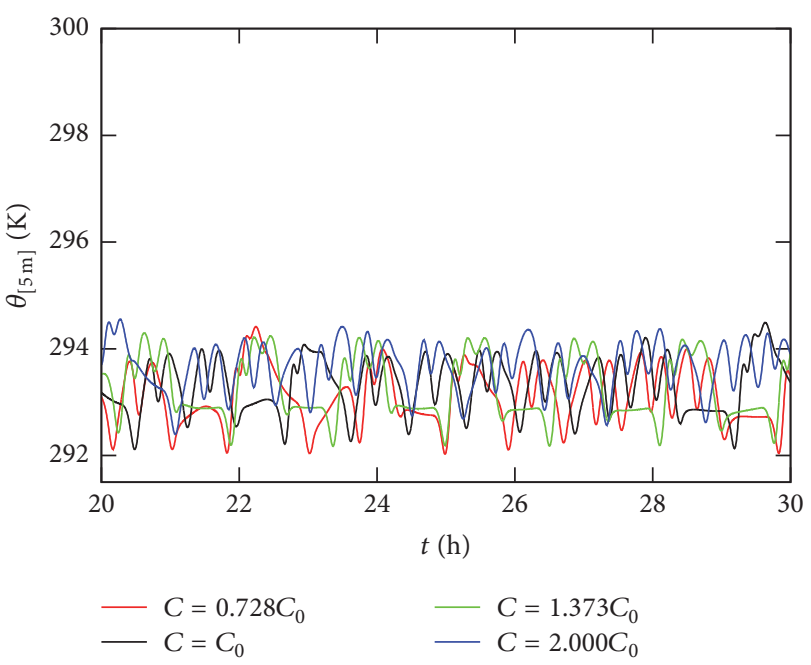

(b) $u_{G}=2.5 \mathrm{~m} \mathrm{~s}^{-1}$

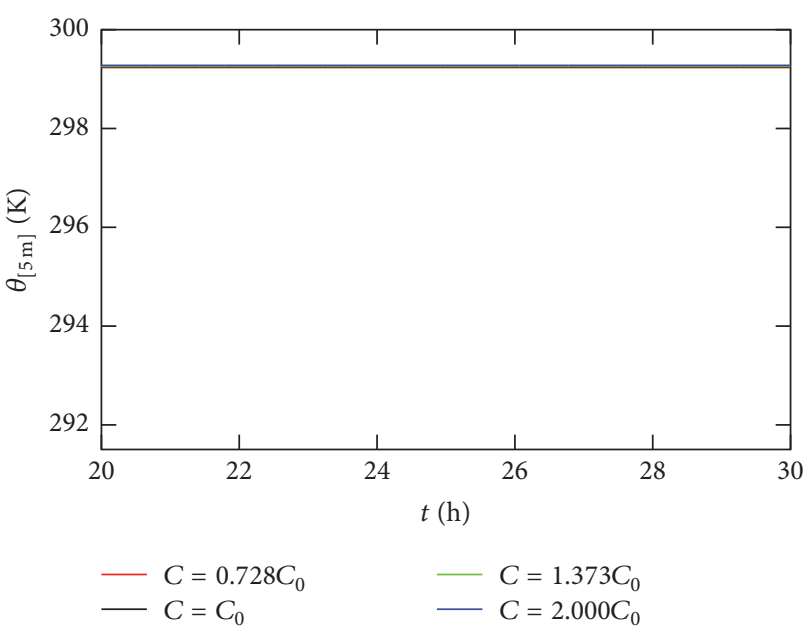

(d) $u_{G}=6.0 \mathrm{~m} \mathrm{~s}^{-1}$

FIGURE 1: Time series of temperature at $5 \mathrm{~m}$, with different atmospheric concentrations of $\mathrm{CO}_{2}$ (indicated in the legend) for different values of the mechanical forcing, in the title of each panel.

difference in the temperatures between the scenarios with clear skies and with $20 \%$ of cloud cover and considering the actual carbon dioxide concentration. It is possible to observe that with light winds the temperature rise can reach $1.3 \mathrm{~K}$. For larger mechanical forcing, but still in the disconnected state, the temperature difference increases and almost reaches $3 \mathrm{~K}$ in the states transition. This occurs due to the system change to the connected state with a smaller value of $u_{G}$, as it is discussed before.

It is important to notice that Figure 5 is not able to provide enough information about the dependence of the temperature difference with the increase of the cloud cover and with variation in the $\mathrm{CO}_{2}$ concentration. Figure 6 considers a carbon dioxide atmospheric concentration that is $73 \%$ of the actual values and shows the analysis of the temperature difference for a cloud cover variation from 0 to $90 \%$ in both connection states. The distinction between the two regimes is clearly observed in the temperature difference map. The disconnected state presents the major temperature differences. In spite of the fact that $\mathrm{CO}_{2}$ concentration is reduced, in the idealized scenario, the radiative contribution of the clouds acts in a determinant way in the nocturnal temperature, mainly in the light wind cases. With clear sky, the incoming longwave radiation is smaller, as the carbon dioxide concentration is lower than the actual one, so the temperature differences are negative. On the other side, as the cloud cover increases, the temperature differences turn quite significantly and may reach $6 \mathrm{~K}$ for winds between 2.5 and $3.0 \mathrm{~m} \mathrm{~s}^{-1}$ and $90 \%$ of cloud cover. The connected state presents, almost totally, temperature differences smaller than $0.5 \mathrm{~K}$ for any cloud cover, if the mechanical forcing is greater than $7 \mathrm{~m} \mathrm{~s}^{-1}$.

If the actual scenario of $\mathrm{CO}_{2}$ concentration is considered, the solution of the model will be sensible just to the cloud cover. As it was already shown by [15], the disconnected state is highly sensible to the cloud cover. When the cloud fraction 


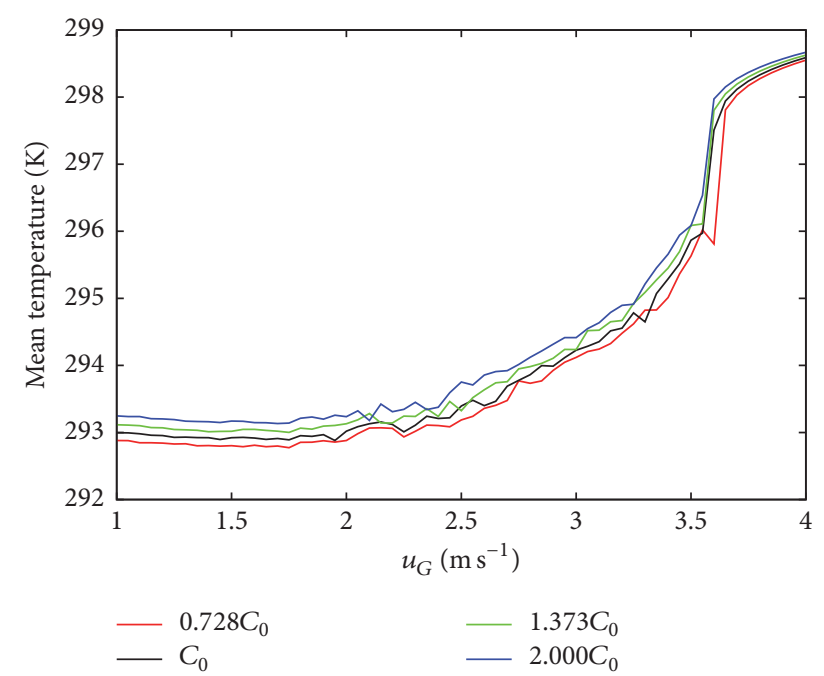

FIgURE 2: Mean temperature at $5 \mathrm{~m}$, for different $\mathrm{CO}_{2}$ concentration (indicated in the legend), as a function of the geostrophic wind.

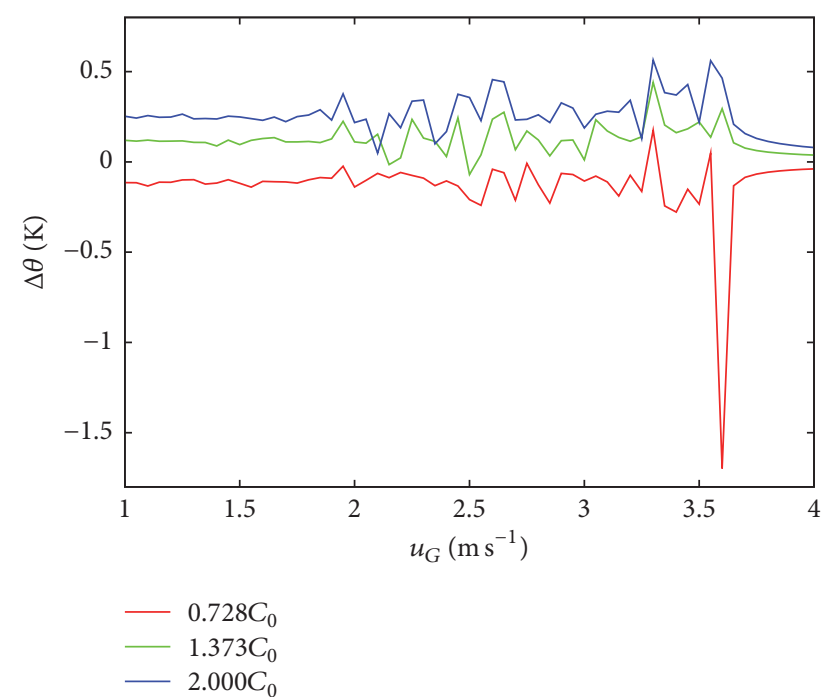

FIgURE 3: Temperature difference between the scenarios with $\mathrm{CO}_{2}$ concentrations (indicated by the legend), in relation to the actual carbon dioxide concentration in the disconnected state.

increases, the incoming longwave radiation increases, and the connection (coupling) can happen at smaller values of the mechanical values.

A hypothetical scenario, where the actual carbon dioxide concentration is doubled, is shown in Figure 7. In general, the temperature difference behavior is held, but the differences are greater than the actual case, as expected. Such temperature difference can reach up to $6.5 \mathrm{~K}$ for $u_{G}$ around $2.5 \mathrm{~m} \mathrm{~s}^{-1}$. In both Figures 6 and 7, it is possible to observe a region where the temperature difference is clearly grater, and it is associated with the regimes transition. The delimitation of this region occurs when $u_{G}$ is around $3.7 \mathrm{~m} \mathrm{~s}^{-1}$, a value for which the transition occurs, and as the cloud cover increases, this region moves to the left in the map due to the connection occurring at smaller values of $u_{G}$.

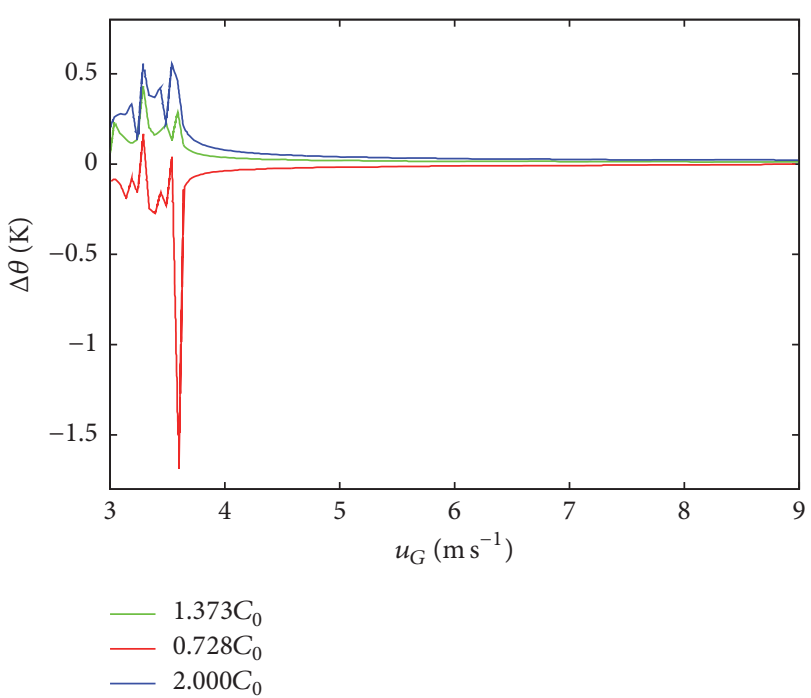

FIgURE 4: Temperature difference between the scenarios with $\mathrm{CO}_{2}$ concentrations (indicated by the legend), in relation to the actual carbon dioxide concentration in the connected state.

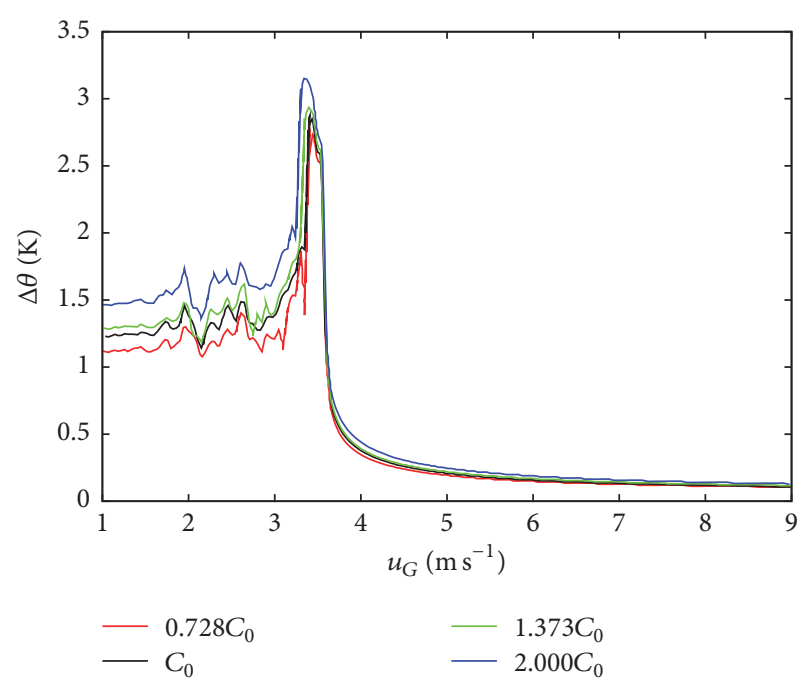

Figure 5: Temperature difference between the scenarios with $20 \%$ of clod covering and clear skies, for different $\mathrm{CO}_{2}$ concentration (indicated in the legend).

\section{Conclusions}

Recent efforts have suggested that the coupling state of the SBL has great influence over the diurnal temperature range (DTR) [12, 13]. However, the role of the intermittent behavior of the nocturnal turbulence on the DTR is not well known. In this work we perform some simple analysis, using the model proposed by Costa [15], for the temperature difference between some scenarios with different carbon dioxide concentration and various cloud cover values. According to [13], the major variability, between the scenarios, occurs in the stable boundary layer (SBL) regime transition.

Although in the decoupled state the flow behavior is unpredictable, the temperature and other scalar variables as 


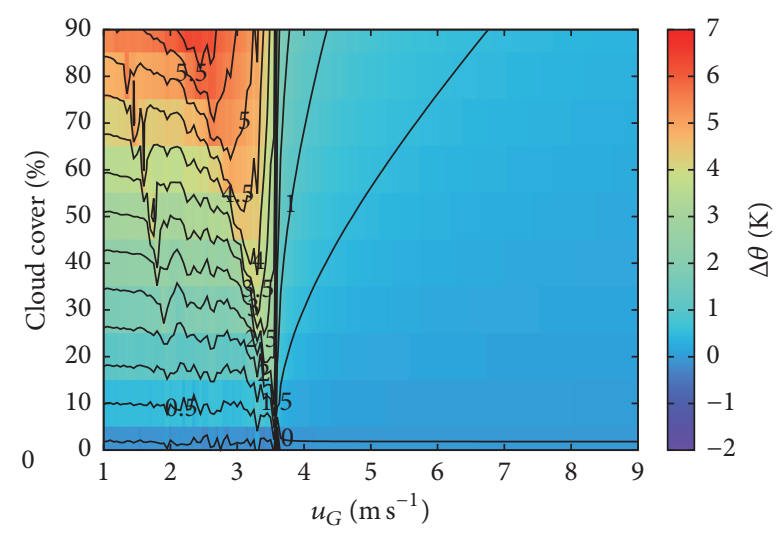

FIGURE 6: Temperature differences considering the variation on the cloud cover from 0 to $90 \%$. The $\mathrm{CO}_{2}$ concentration is considered being $73 \%$ of the actual value.

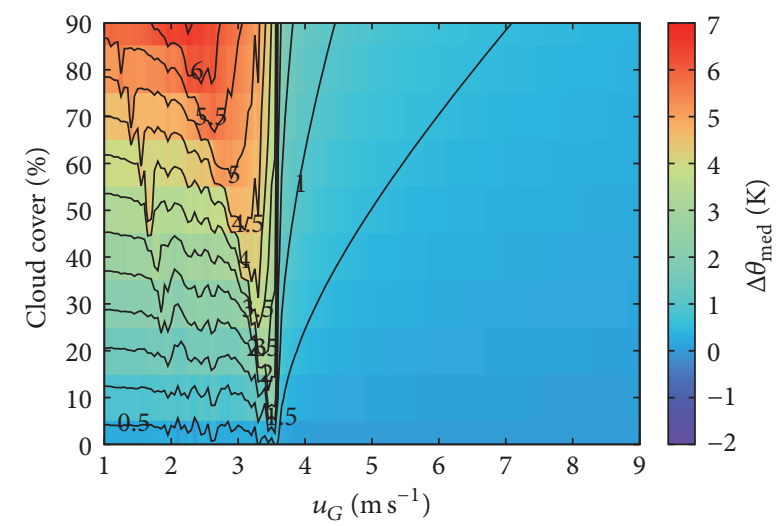

FIGURE 7: Temperature differences considering the variation on the cloud cover from 0 to $90 \%$. The $\mathrm{CO}_{2}$ concentration is considered being double the actual value.

contaminants are also affected by this complexity. Reference [12] has shown that for light winds the turbulence intensity is not enough to dissipate or transport the internal energy in excess in the lower atmosphere to upper levels, and because of this the temperature tends to rise in very stable conditions when the radiative forcing increases. Here, we show that, beyond the increase caused even by the $\mathrm{CO}_{2}$ concentration in the atmosphere or for the cloud cover, the temperature variation can be unpredictable in the disconnected state due to the occurrence of the intermittent bursts.

Another important point is that the temperature difference in the disconnected state rises as the wind increases. This happens in virtue of the intensification of the turbulent bursts which are responsible for bringing the warm air from the upper levels of the atmosphere down, as well as carrying the cold air, which lies near the surface, until the higher SBL levels. In this way, it is impossible to predict a tendency for the mean temperature, when just the $\mathrm{CO}_{2}$ is changed.

In the transition region between the two regimes of connection the temperature difference between the scenarios becomes more intense. This occurs due the connection being highly dependent on the energy supplement that is available for the system. So, the greater the amount of internal energy is, the greater the radiative forcing is and the smaller the value of $u_{G}$ necessary to the connection will be, which leads to a bigger temperature difference in relation to the actual scenario. This result indicates that, for example, if a hypothetical region is characterized by moderate winds in the nocturnal period, its DTR could be drastically reduced if the radiative forcing is large enough to change the coupling state in this region, while, in regions characterized by moderate to strong winds, the temperature variations will be reduced due to the strong turbulence activity [12].

The results also show that as the cloud cover increases, the system tends to keep being connected, as the incoming longwave radiation is major, and it is enough to maintain the model connected even with moderate winds.

Finally, it is important to stress that the analysis shown here is based on a theoretical simplified model and in spite of being in agreement with previous efforts and to present the role of the intermittency in the temperature variations, it is necessary to validate the analysis done here using real data. Besides, a possible further improvement in the model for future studies is to include radiative flux divergence in the energy equation.

\section{Conflicts of Interest}

The authors declare that they have no conflicts of interest.

\section{Acknowledgments}

This work was supported by Brazilian Research Agencies CNPq (Conselho Nacional de Desenvolvimento Científico e Tecnológico), CAPES (Comissão de Pessoal de Aperfeiçoamento de Pessoal de Ensino Superior), and FAPERGS (Fundação de Amparo à Pesquisa do Estado do Rio Grande do Sul). The simulations were performed in a computer system funded by the program FAPERGS-Pesquisador Gaúcho 2014 (Process 2312-2551/14-0).

\section{References}

[1] J. T. Houghton et al., "Climate change 2001: the scientific basis," The Press Syndicate of the University of Cambridge.

[2] R. S. Vose, D. R. Easterling, and B. Gleason, "Maximum and minimum temperature trends for the globe: an update through 2004," Geophysical Research Letters, vol. 32, no. 23, 2005.

[3] K. Makowski, M. Wild, and A. Ohmura, "Diurnal temperature range over Europe between 1950 and 2005," Atmospheric Chemistry and Physics, vol. 8, no. 21, pp. 6483-6498, 2008.

[4] H. X. Cao, J. F. B. Mitchell, and J. R. Lavery, "Simulated diurnal range and variability of surface temperature in a global climate model for present and doubled CO2 climates," Journal of Climate, vol. 5, no. 9, pp. 920-943, 1992.

[5] D. Stone and A. Weaver, "Factors contributing to diurnal temperature range trends in twentieth and twenty-first century simulations of the CCCma coupled model," Climate Dynamics, vol. 20, no. 5, pp. 435-445, 2003.

[6] L. Mahrt, "Stratified atmospheric boundary layers," BoundaryLayer Meteorology, vol. 90, no. 3, pp. 375-396, 1999. 
[7] S. H. Derbyshire, "Boundary-layer decoupling over cold surfaces as a physical boundary-instability," Boundary-Layer Meteorology, vol. 90, no. 2, pp. 297-325, 1999.

[8] J. Sun, L. Mahrt, R. M. Banta, and Y. L. Pichugina, "Turbulence regimes and turbulence intermittency in the stable boundary layer: During CASES-99," Journal of the Atmospheric Sciences, vol. 69, no. 1, pp. 338-351, 2012.

[9] O. C. Acevedo, L. Mahrt, F. S. Puhales, F. D. Costa, L. E. Medeiros, and G. A. Degrazia, "Contrasting structures between the decoupled and coupled states of the stable boundary layer," Quarterly Journal of the Royal Meteorological Society, vol. 142, no. 695, pp. 693-702, 2016.

[10] B. J. H. Van de Wiel, A. F. Moene, H. J. J. Jonker et al., "The minimum wind speed for sustainable turbulence in the nocturnal boundary layer," Journal of the Atmospheric Sciences, vol. 69, no. 11, pp. 3116-3127, 2012.

[11] O. C. Acevedo and D. R. Fitzjarrald, "In the core of the nightEffects of intermittent mixing on a horizontally heterogeneous surface," Boundary-Layer Meteorology, vol. 106, no. 1, pp. 1-33, 2003.

[12] R. T. McNider, G. J. Steeneveld, A. A. Holtslag et al., "Response and sensitivity of the nocturnal boundary layer over land to added longwave radiative forcing," Journal of Geophysical Research: Atmospheres, vol. 117, no. D14, 2012.

[13] J. T. Walters, R. T. McNider, X. Shi, and W. B. Norris, "Positive surface temperature feedback in the stable nocturnal boundary layer," Geophysical Research Letters, vol. 34, no. 12, Article ID L12709, 2007.

[14] R. T. McNider, D. E. England, M. . Friedman, and X. Z. Shi, "Predictability of the stable atmospheric boundary layer," Journal of the Atmospheric Sciences, vol. 52, no. 10, pp. 1602-1614, 1995.

[15] F. D. Costa, O. C. Acevedo, J. C. M. Mombach, and G. A. Degrazia, "A simplified model for intermittent turbulence in the nocturnal boundary layer," Journal of the Atmospheric Sciences, vol. 68, no. 8, pp. 1714-1729, 2011.

[16] J. R. Garratt and R. A. Brost, "Radiative cooling effects within and above the nocturnal boundary layer," Journal of the Atmospheric Sciences, vol. 38, no. 12, pp. 2730-2746, 1981.

[17] J. M. Edwards, "Radiative processes in the stable boundary layer: Part II. The development of the nocturnal boundary layer," Boundary-Layer Meteorology, vol. 131, no. 2, pp. 127-146, 2009.

[18] A. K. Blackadar, "High resolution models of the planetary boundary layer," Advances in Environmental Science And Engineering, vol. 1, no. 1, pp. 50-85, 1979.

[19] E. F. Harrison, P. Minnis, B. R. Barkstrom, V. Ramanathan, R. D. Cess, and G. G. Gibson, "Seasonal variation of cloud radiative forcing derived from the earth radiation budget experiment," Journal of Geophysical Research: Atmospheres, vol. 95, no. D11, pp. 18687-18703, 1990.

[20] D. O. Staley and G. M. Jurica, "Effective atmospheric emissivity under clear skies," Journal of Applied Meteorology, vol. 11, no. 2, pp. 349-356, 1972.

[21] J. T. Houghton, G. J. Jenkins, and J. J. Ephraums, Report prepared for intergovernmental panel on climate change by working group I.

[22] P. G. Duynkerke, "Application of the E- $\varepsilon$ turbulence closure model to the neutral and stable atmospheric boundary layer," Journal of the Atmospheric Sciences, vol. 45, no. 5, pp. 865-880, 1988.
[23] W. Weng and P. A. Taylor, "On modelling the one-dimensional atmospheric boundary layer," Boundary-Layer Meteorology, vol. 107, no. 2, pp. 371-400, 2003.

[24] H. A. Panofsky and J. A. Dutton, "Atmospheric turbulence: models and methods for engineering applications," 397 pages, 1984.

[25] R. A. Pielke SR, Mesoscale meteorological modeling, Academic press, 2013.

[26] A. N. Kolmogoroff, "The local structure of turbulence in incompressible viscous fluid for very large Reynold's numbers," pp. 301-305, 1941.

[27] A. N. Kolmogoroff, "Dissipation of energy in the locally isotropic turbulence," pp. 16-18, 1941.

[28] J. Cuxart, A. A. M. Holtslag, R. J. Beare et al., "Single-column model intercomparison for a stably stratified atmospheric boundary layer," Boundary-Layer Meteorology, vol. 118, no. 2, pp. 273-303, 2006.

[29] G. L. Mellor and T. Yamada, "A hierarchy of turbulence closure models for planetary boundary layers," Journal of the Atmospheric Sciences, vol. 31, no. 7, pp. 1791-1806, 1974.

[30] D. O. Revelle, "Chaos and "bursting' in the planetary boundary layer," Journal of Applied Meteorology, vol. 32, no. 7, pp. 11691180, 1993.

[31] B. J. H. Van de Wiel, R. J. Ronda, A. F. Moene, H. A. R. De Bruin, and A. A. M. Holtslag, "Intermittent turbulence and oscillations in the stable boundary layer over land. Part I: A bulk model," Journal of the Atmospheric Sciences, vol. 59, no. 5, pp. 942-958, 2002.

[32] L. Zhou, R. E. Dickinson, A. Dai, and P. Dirmeyer, "Detection and attribution of anthropogenic forcing to diurnal temperature range changes from 1950 to 1999: Comparing multi-model simulations with observations," Climate Dynamics, vol. 35, no. 7, pp. 1289-1307, 2010.

[33] A. Dai, K. E. Trenberth, and T. Qian, "A global dataset of Palmer Drought Severity Index for 1870-2002: relationship with soil moisture and effects of surface warming," Journal of Hydrometeorology, vol. 5, no. 6, pp. 1117-1130, 2004. 

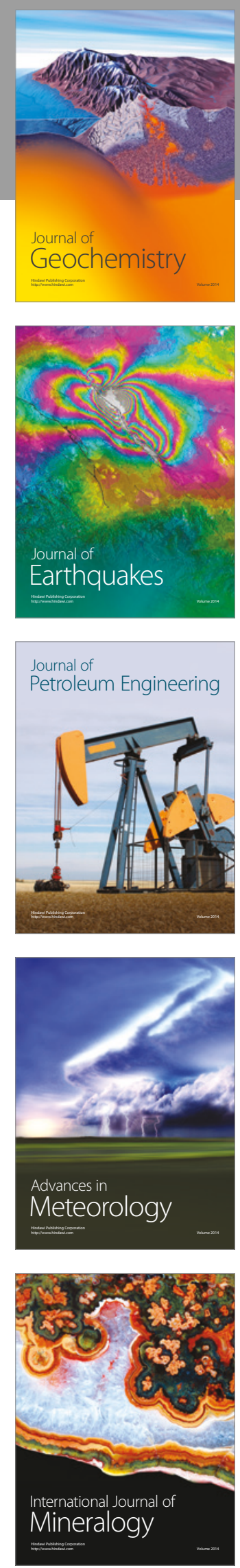
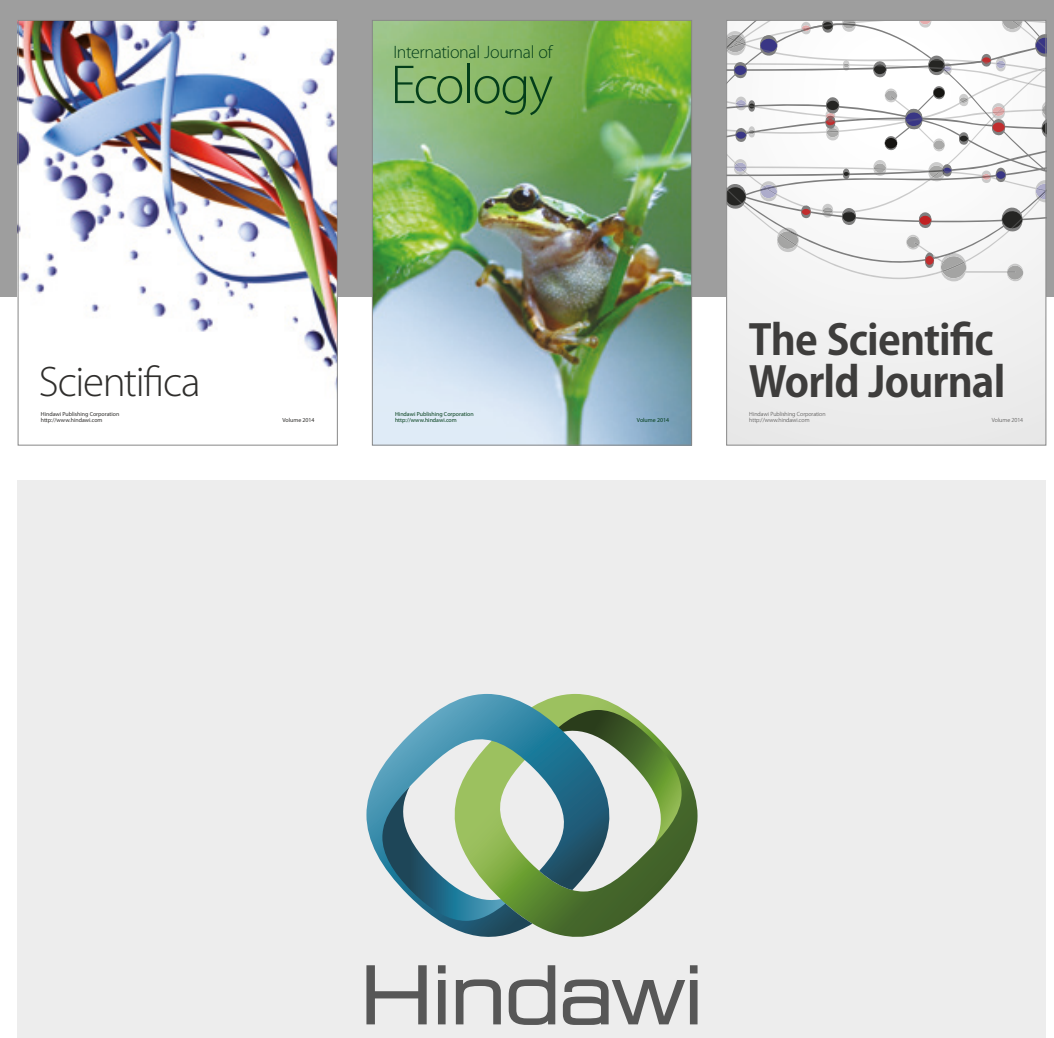

Submit your manuscripts at

https://www.hindawi.com
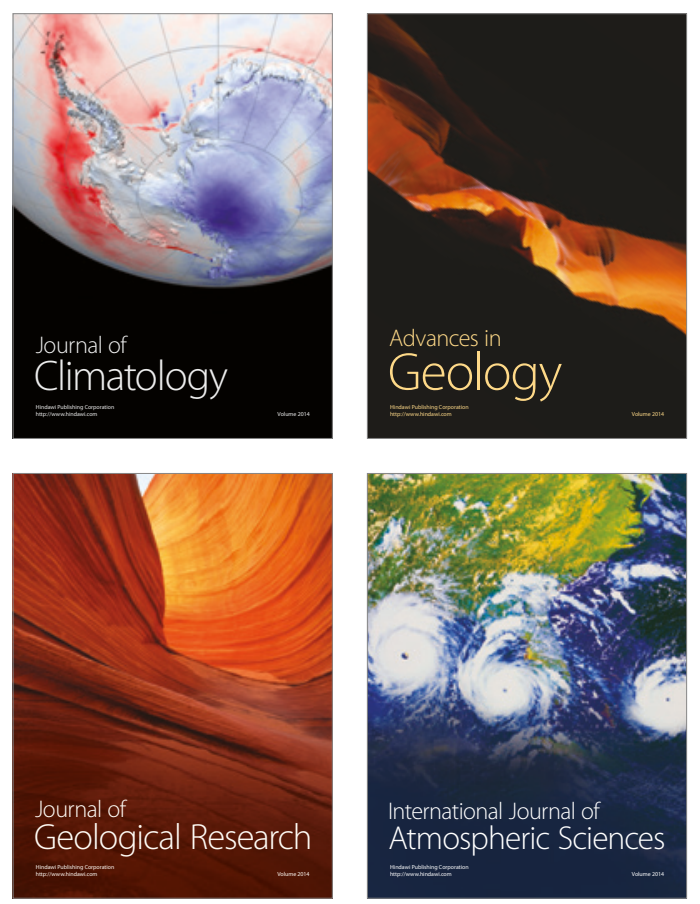

The Scientific

World Journal
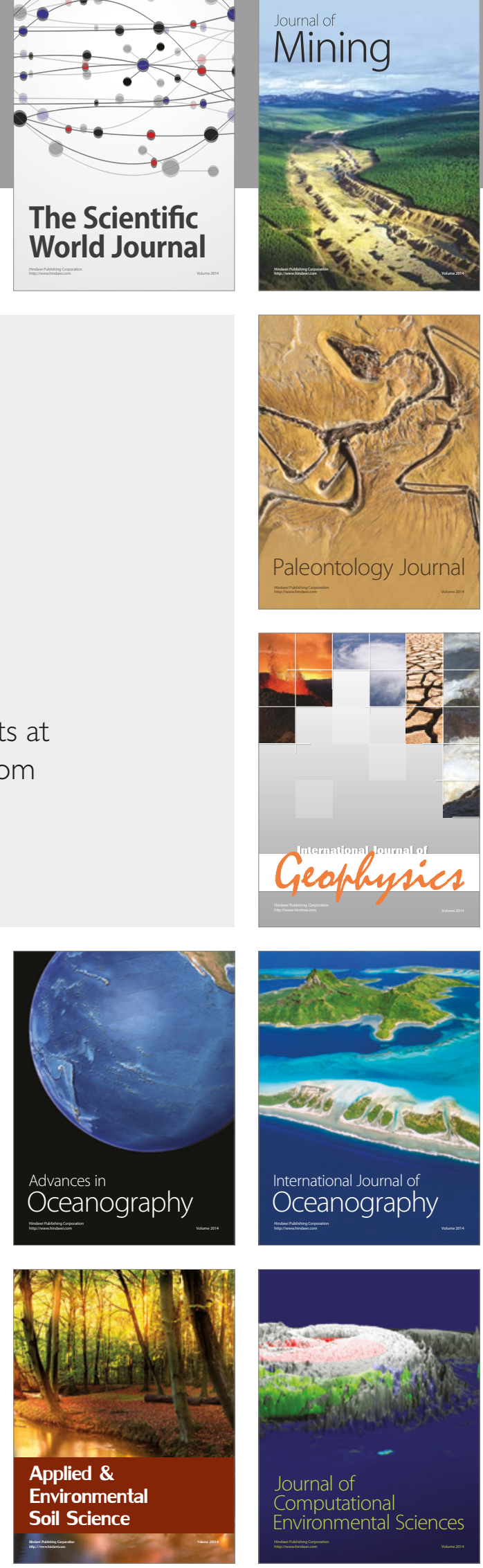